\title{
JEJUNO BILIAR: OBSTRUÇÃO INTESTINAL POR CÁLCULO BILIAR EM UM PACIENTE IDOSO COM VÍCIO DE ROTAÇÃO
}

\section{GALLSTONE JEJUNUM: INTESTINAL OBSTRUCTION DUE TO GALLSTONES IN AN ELDERLY PA- TIENT WITH INTESTINAL MALROTATION}

Bruno Henrique Nunes Hirata ${ }^{1}$; Pedro Chiaramelli ${ }^{1}$; Caio Nasser Mancini ${ }^{1}$; Marcos Henrique de Andrade Zanoni ${ }^{1}$; Lucas Moreto Betini ${ }^{1}$; Henrique Cunha Mateus ${ }^{1}$.

\section{RESUMO}

Introdução: O îleo biliar corresponde de 1 a $4 \%$ das causas de obstruções intestinais. Trata-se de uma complicação rara da colelitíase não tratada, decorrente de uma complicação da colecistite aguda: uma fistula biliodigestiva. Anomalias congênitas da rotação intestinal também podem ser causas de obstrução intestinal e, em adultos, sua incidência está descrita em aproximadamente $0,2 \%$ dos casos. Relato do caso: Relatamos um caso incomum de um paciente com aerobilia, obstrução intestinal por cálculo biliar (diagnosticados na tomografia de abdome). Na laparotomia exploradora, foi constatada a presença de um vício de rotação com Banda de Ladd. Foi retirado o cálculo do jejuno e não foi abordada a fistula biliar. O paciente evoluiu com uma broncopneumonia e faleceu no terceiro dia pós-operatório. Conclusão: Em suma, o íleo biliar é uma das complicações da colelitíase não tratada que necessita de intervenção de urgência. Seu diagnóstico requer um alto grau de suspeição e seu tratamento cirúrgico deve ser imediato. A Banda de Ladd é uma causa de obstrução duodenal em crianças com vício de rotação intestinal. Seu diagnóstico em adultos é pouco frequente. O paciente supracitado apresentava duas doenças raras simultâneas.

Palavras-chave: Colelitíase. Obstrução Intestinal. Jejuno. Laparotomia. Fistula do Sistema Digestório.

\section{ABSTRACT}

Introduction: The bile ileum corresponds to 1 to $4 \%$ of the causes of intestinal obstructions. It is a rare complication of untreated cholelithiasis, resulting from a complication of acute cholecystitis: a biliodigestive fistula. Congenital anomalies of intestinal rotation can also be causes of intestinal obstruction and, in adults, its incidence is described in approximately $0.2 \%$ of cases. Case report: We report an unusual case of a patient with aerobilia, intestinal obstruction due to gallstones (diagnosed on abdominal tomography). In exploratory laparotomy, the presence of a rotation defect with the Ladd Band was found. The jejunum stone was removed and biliary fistula was not addressed. The patient developed bronchopneumonia and died on the third postoperative day. Conclusion: In short, bile ileus is one of the complications of untreated cholelithiasis that requires urgent intervention. Its diagnosis requires a high degree of suspicion and its surgical treatment must be immediate. Ladd Band is a cause of duodenal obstruction in children with addiction to intestinal rotation. Its diagnosis in adults is uncommon. The aforementioned patient had two rare simultaneous diseases.

Keywords: Cholelithiasis. Intestinal Obstruction. Jejunum. Laparotomy. Biliary Fistula.

\section{INTRODUÇÃO}

A colelitíase é uma doença de grande prevalência. Circunscrita nas patologias do aparelho digestivo, ela é a principal causa de admissão em países desenvolvidos e, portanto, demanda uma parte importante nos gastos de saúde ${ }^{1,2}$. Cerca de $10 \%$ da população adulta do Reino Unido e 10\% a $15 \%$ dos Estados Unidos possuem cálculos biliares ${ }^{1,3}$. No Brasil, a prevalência é similar: aproximadamente $10 \%$ dos brasileiros possuem litíase biliar4,5. Sua incidência está relacionada à progressão da idade. Dessa forma, a prevalência de 10\% chega a $21 \%$ na população de 60 a 69 anos e mais de $30 \%$ nos indivíduos acima de 70 $\operatorname{anos}^{6}$.

Clinicamente, essa doença pode-se apresentar em três formas: assintomática, sintomática não-complicada e sintomática complicada. A primeira é mais predominante, sendo encontrada 
acidentalmente em exames de imagem (ultrassonografia ou tomografia computadorizada). Entretanto, cerca de $2 \%$ dos doentes podem desenvolver sintomas ou apresentar alguma complicação ${ }^{6}$. Os sintomas mais encontrados na colelitíase são dor em hipocôndrio direito e icterícia. Entre as formas complicadas, destacam-se a colecistite aguda, a pancreatite aguda biliar e a coledocolitíase. A formação de fístula biliodigestiva, com passagem de cálculos para o trato digestivo, é uma condição que ocorre em raros casos e pode obstruir algum segmento intestinal, dependendo do tamanho do cálculo e do local de formação da fistula. Geralmente, essa obstrução ocorre na porção distal do íleo, na válvula ileocecal. Tal condição é denominada íleo biliar.

Anomalias congênitas da rotação intestinal também podem ser causas de obstrução intestinal e são encontradas em apenas um a cada 500 nascidos vivos. A maioria dos casos é diagnosticada no período neonatal, podendo ser complicada para volvos e isquemia intestinal 7 . Em adultos, é uma malformação de diagnóstico extremamente raro, sendo diagnosticada em apenas $0,2 \%$ dos casos. Ela pode se manifestar como causa de abdome agudo obstrutivo ou, quando crônico, como uma dor abdominal intermitente ou permanecer assintomático durante toda a vida ${ }^{7,8}$. O presente trabalho visa compartilhar um raro caso de íleo biliar em idoso com Banda de Ladd e seu tratamento cirúrgico.

\section{RELATO DO CASO}

Paciente de sexo masculino, de 86 anos, com história prévia de hipertensão arterial sistêmica, tabagista com carga tabágica de 60 anos-maço e sem cirurgias prévias deu entrada no serviço de emergência do Hospital São Luiz Gonzaga, no Estado de São Paulo, Brasil, com quadro de dor abdominal há quatro dias, de inicio súbito, difusa, sem irradiação, em cólica, de moderada intensidade, constante e sem fatores de melhora e piora. Há um dia, passou a apresentar náuseas e vômitos. Relatou parada de evacuação no período descrito, mas não parou de eliminar gases. Ao exame físico, paciente apresentava em mau estado geral, estava consciente, contactuando, orientado, eupneico, desidratado $(2+/ 4+)$, estava descorado $(1+/ 4+)$. Possuía frequência cardíaca de
$110 \mathrm{bpm}$, perfusão periférica de $2 \mathrm{~s}$ e pressão arterial de $110 \times 70 \mathrm{mmHg}$. No exame físico, seu abdome se apresentava distendido, timpânico, doloroso difusamente, mas sem sinais de peritonite. Ao toque retal, havia fezes, não havia tumor tocável, nem sangue.

Com as informações supracitadas, chegou-se a hipótese diagnóstica de abdome agudo obstrutivo. Foram realizadas a hidratação do paciente, a passagem de uma sonda nasogástrica com retirada de $1.700 \mathrm{~mL}$ de volume, radiografia de abdome e exames laboratoriais. A radiografia do abdome mostrou somente velamento, não sendo possivel visualizar distensão de alças intestinais. Os exames laboratoriais evidenciaram leucocitose sem desvio, alterações dos niveis de uréia e creatinina e alcalose metabólica.

Foi realizada tomografia computadorizada do tórax e abdome (Figuras 1 e 2), que definiu o que seria na radiografia a tríade de Rigler (oclusão intestinal, litíase biliar ectópica e aerobilia). Paciente foi encaminhado ao centro cirúrgico com o diagnóstico de íleo biliar.

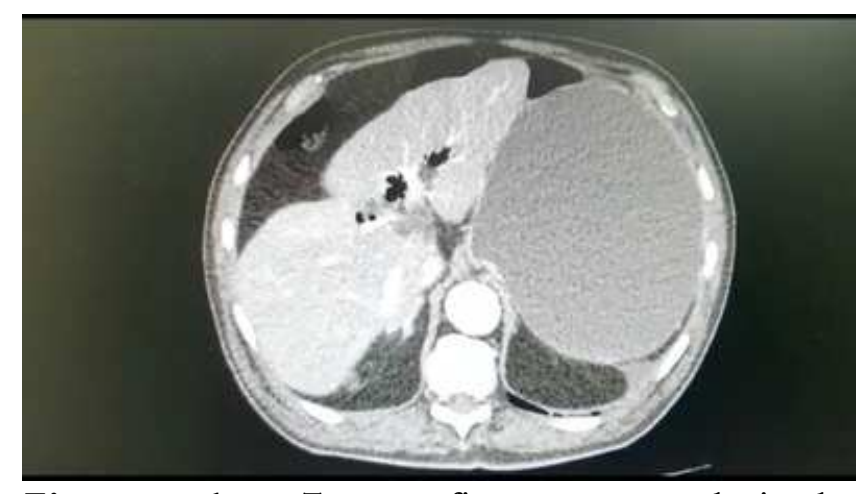

Figura 1. Tomografia computadorizada evidenciando oclusão intestinal, litíase biliar ectópica e aerobilia.

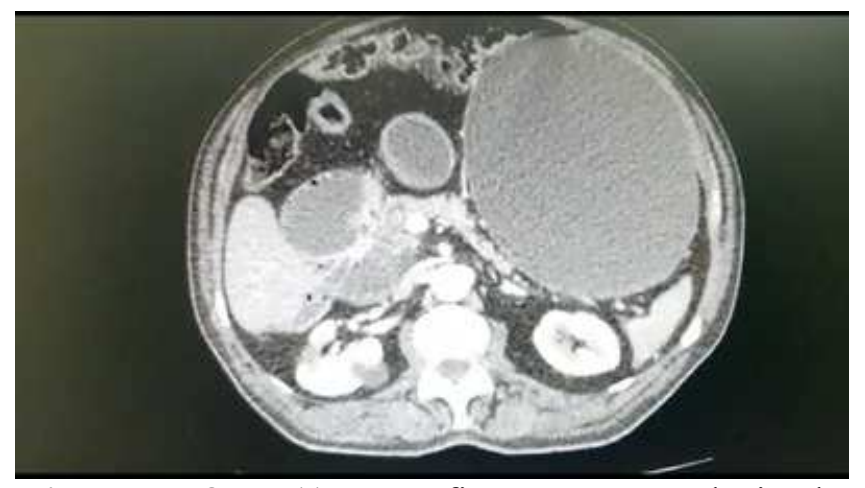

Figura 2. Tomografia computadorizada evidenciando oclusão intestinal, litíase biliar ectópica e aerobilia. 
Com o paciente sob anestesia geral e posicionado em decúbito dorsal, foi realizada a laparotomia com incisão mediana. $\mathrm{Na}$ inspeção da cavidade abdominal, não havia líquido livre, notou-se bloqueio do hipocôndrio direito; distensão do estômago, duodeno e delgado por $60 \mathrm{~cm}$ onde se palpava estrutura oval de cerca de $5 \mathrm{~cm} \times 3 \mathrm{~cm}$. Ademais, foi observado um vício de rotação, com duodeno retificado nas $2^{\mathrm{a}}, 3^{\mathrm{a}}$ e $4^{\mathrm{a}}$ porções, lateral ao cólon direito que era fixo à parede abdominal pela Banda de Ladd (Figura 3).

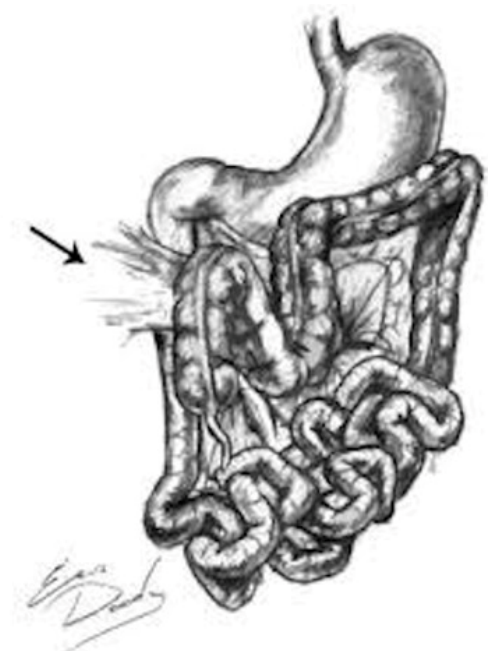

Figura 3. Representação da Banda de Ladd. Fonte: D. Nehra and A. M. Goldstein, "Intestinal malrotation: Varied clinical presentation from infancy through adulthood"9

O referido bloqueio no hipocôndrio direito não foi abordado. Foi realizada uma enterotomia longitudinal proximal ao ponto de obstrução, mobilizando o cálculo até a enterotomia e o retirando (Figuras 4 e 5). O tempo da cirurgia foi de 55 minutos.

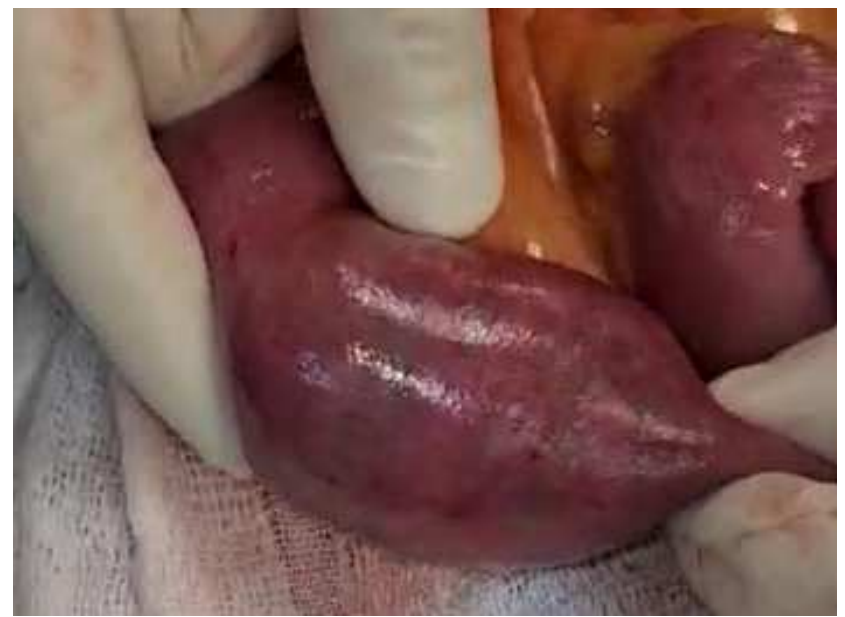

Figura 4. Mobilização do cálculo e sua retirada pela enterolitotomia.

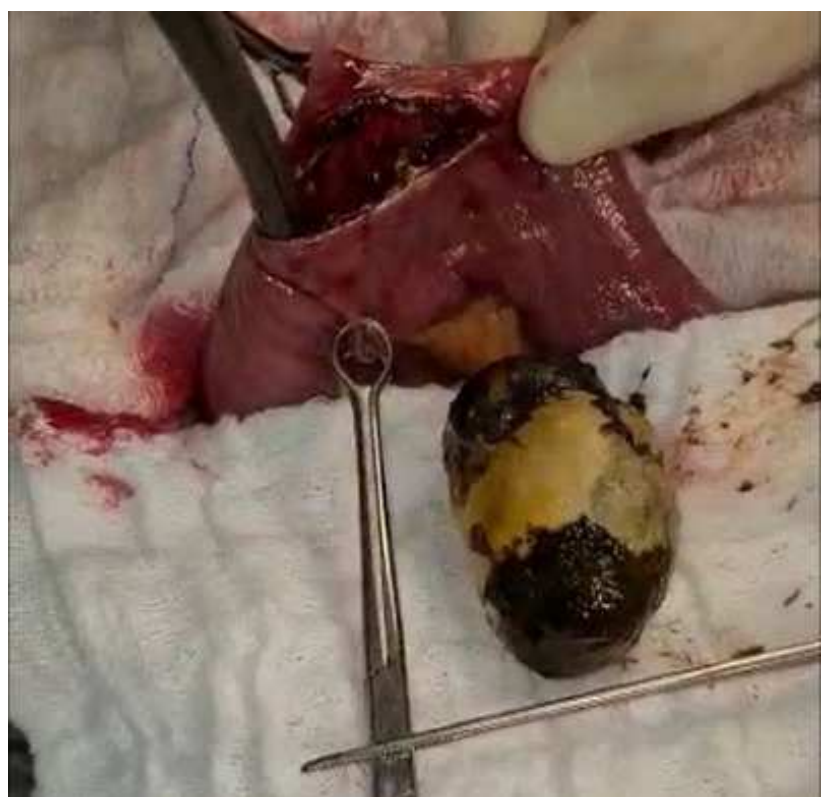

Figura 5. Mobilização do cálculo e sua retirada pela enterolitotomia.

O paciente foi encaminhado para unidade de terapia intensiva (UTI) após o procedimento com necessidade de droga vasoativa e sob ventilação mecânica. No primeiro dia pós-operatório, realizou radiografia de tórax que apontou broncopneumonia aspirativa. Apesar do suporte clínico, faleceu no terceiro dia pósoperatório.

\section{DISCUSSÃO}

O íleo biliar foi descrito pela primeira vez por Bartholin, em 1654, e é responsável por até $25 \%$ das obstruções do intestino delgado em pacientes com mais de 65 anos, com preponderância feminina ${ }^{10}$. Trata-se de uma complicação que acomete apenas $0,3 \%$ a $0,5 \%$ dos pacientes com colelitíase. $O$ cálculo biliar obstrui a luz intestinal através de uma fistula biliodigestiva 6 . A fistula biliodigestiva é formada da erosão provocada pelo processo inflamatório da colecistite e aderência entre a vesícula biliar e o trato digestivo. Pode-se dizer que há maior predileção anatômica para a formação das fistulas no duodeno, pois entre elas, a colecistoduodenal corresponde a mais de $70 \%$ dos casos. Entretanto, há casos de formação de fistulas biliodigestivas em qualquer lugar do trato digestivo, incluindo estômago, cólon e outras regiões do intestino delgado6-10. Quando o cálculo atravessa a fistula, a depender de sua localização, ele pode ser eliminado pelas fezes, ficar impactado em algum segmento 
do sistema digestivo ou ser expelido pelo vômito11. Caso ele fique impactado na válvula íleo-cecal, o quadro obstrutivo é denominado íleo biliar. Kasahara et al. relataram que apenas 8 dos 112 pacientes com îleo biliar tiveram eliminação espontânea dos cálculos ${ }^{12}$. O sítio de obstrução pode ser localizado em qualquer lugar do trato gastrointestinal, porém a porção terminal do íleo, na válvula ileocecal, é o ponto mais frequente de obstrução ${ }^{6}$. Reisner et al. realizaram uma revisão com 1.001 casos reportados $\mathrm{e}$ evidenciaram que o cálculo impactado no cólon representava apenas $4 \%$ dos pacientes. O diagnóstico do íleo biliar se baseia no quadro clínico e exames de imagem. De modo geral, o paciente é diagnosticado com quadro de abdome agudo obstrutivo. A suspeita do íleo biliar aumenta quando se nota a aerobilia e cálculos impactados no trânsito intestinal ${ }^{6}$. Ayantunde et al. realizaram um estudo com 22 pacientes com média de 77 anos e predominio do sexo feminino (18 mulheres). A maioria dos pacientes apresentaram dor abdominal (100\%) e vômitos $(95,5 \%)$, com cerca de 3 dias de duração dos sintomas. O diagnóstico pré-operatório foi realizado em $77 \%$ dos casos, com o auxílio de exames de imagem (ultrassonografia, radiografia e tomografia computadorizada). Em cerca de $15 \%$ a $50 \%$ dos casos, é possivel observar a tríade de Rigler nos exames de imagem, a qual consiste na oclusão intestinal, cálculo biliar ectópica e aerobilia10,13. Entretanto, muitas vezes o diagnóstico é realizado durante a laparotomia, devido ao seu baixo grau de suspeição. $O$ tratamento cirúrgico do íleo biliar consiste na enterolitotomia, colecistectomia e a correção da fístula biliodigestiva. Entretanto, não há consenso na literatura quanto à realização da colecistectomia e exploração da fístula no mesmo momento da enterolitotomia6,10,14-46. É necessário avaliar o grau de obstrução do trânsito intestinal, a idade do paciente, os achados locais e comorbidades. Reisner et al. concluíram que o tratamento da fistula simultâneo ao da obstrução está associado a uma mortalidade de $16,9 \%$, enquanto a enterolitotomia isolada, $11,7 \%$. Logo, a escolha da colecistectomia e correção da fistula concomitantemente à enterotomia deve estar reservada para pacientes altamente selecionados sob o risco de ter que ser feita uma gastrectomia em um paciente com obstrução de intestino delgado. Anomalias congênitas da rotação intestinal também podem ser causas de obstrução intestinal. A maioria dos casos é diagnosticada no período neonatal, que pode complicar para os volvos e isquemia intestinal7. Em adultos, é uma malformação extremamente rara, sendo diagnosticada em aproximadamente 0,2\% dos casos. Entretanto, trata-se de uma doença de dificil precisão epidemiológica. Estudos pós mortem sugerem uma incidência de malformação intestinal de até um a cada seis mil8,17. Elas podem se manifestar como causa de abdome agudo obstrutivo ou, quando crônico, como uma dor abdominal intermitente ou permanecer assintomático durante toda a vida ${ }^{7,8}$. A maioria dos diagnósticos de vício de rotação intestinal em adultos é feita em pacientes assintomáticos e é encontrado incidentalmente por exames de imagem ou como achado incidental durante uma cirurgia8 ${ }^{8}$ O tratamento cirúrgico da má rotação intestinal foi descrito pela primeira vez por William Ladd em 1936 e este procedimento cirúrgico continua sendo a base do tratamento. $O$ procedimento de Ladd consiste em 4 partes: divisão das bandas Ladd, recobrindo o duodeno; alargamento da raiz estreita do mesentério do intestino delgado, mobilizando o duodeno e divisão das adesões em torno da artéria mesentérica superior para prevenir o volvo; distorção anti-horária do volvo de intestino, se presente, e apendicectomia para prevenir um futuro diagnóstico de um apêndice anormalmente localizado ${ }^{18}$. Assim como o manejo do íleo biliar, o tratamento cirúrgico para pacientes assintomáticos com vício de rotação intestinal é controverso. Choi et al. revisaram 177 pacientes em um estudo retrospectivo e observaram que a maioria dos casos assintomáticos tinham baixo risco para as complicações de volvo e concluíram que cirurgias eletivas do procedimento de Ladd para esses casos era contraindicada ${ }^{19}$. Entretanto, há na literatura a defesa da correção do vício de rotação intestinal independentemente da idade e da presença dos sintomas, pois não há como prever a manifestação das graves complicações desta condição ${ }^{7}$. 


\section{CONCLUSÃO}

O íleo biliar é uma das complicações da colelitíase não tratada que podem ser graves, necessitando de intervenções de urgência. Trata-se de uma doença de dificil diagnóstico. Os exames de imagem auxiliam na determinação da doença, possibilitando uma melhor acurácia e menor tempo de investigação clínica. Seu diagnóstico requer um alto grau de suspeição. O tratamento cirúrgico consiste na enterolitotomia, colecistectomia e correção da fístula biliodigestiva e pode ser aplicado no mesmo momento apenas em pacientes com baixo risco operatório. A má rotação intestinal é uma doença extremamente rara em adultos. A maioria dos casos desta comorbidade é assintomática e seu diagnóstico é predominantemente incidental por exames de imagem e como achado incidental durante uma cirurgia. Seu tratamento é baseado pela cirurgia descrita por Ladd e sua realização em assintomáticos ainda é questionada.

\section{REFERÊNCIAS}

1. Beckingham IJ. ABC of diseases of liver, pancreas, and biliary system: Gallstone disease. BMJ. 2001;322(7278):91-4. doi: $10.1136 /$ bmj.322.7278.91.

2. Stinton LM, Myers RP, Shaffer, EA. Epidemiology of gallstones. Gastroenterol Clin North Am. 2010:39(2):15769.

doi: 10.1016/j.gtc.2010.02.003.

3. Clavien PA, Richon J, Burgan S, Rohner A. Gallstone ileus. $\mathrm{Br} J$ Surg. 1991;77(7):737-42.

doi: $10.1002 /$ bjs. 1800770707 .

4. Coelho JC, Bonilha R, Pitaki SA, Cordeiro RM, Salvalaggio PR, Bonin EA, et al. Prevalence of gallstones in a Brazilian population. Int Surg. 1999;84(1):25-8.

5. Graciano AR, Squeff FA. Epidemiological profile of cholelithiasis in Brazil: 10-year analysis. Rev. Educ. Saúde. 2019;7(2):111-7. doi: 10.29237/23589868.2019v7i2.p109-115.

6. Guimarães S, Moura JC, Pacheco Jr, Silva Rodrigo A. Íleo biliar - uma complicação da doença calculosa da vesícula biliar. Rev. bras. geriatr. gerontol. 2010;13(1):159-63. doi: $10.1590 / \mathrm{S} 1809-98232010000100017$.

7. Emanuwa OF, Ayantunde AA, Davies TW. Midgut malrotation first presenting as acute bowel obstruction in adulthood: a case report and literature review. World J Emerg Surg. $2011 ; 6(1): 22$.

doi: 10.1186/1749-7922-6-22.

8. von Flüe $M$, Herzog U, Ackermann C, Tondelli P, Harder F. Acute and chronic presentation of intestinal nonrotation in adults. Dis Colon Rectum. 1994;37(2):192-8.

doi: $10.1007 /$ BF02047549.

9. Nehra D, Goldstein A. Intestinal malrotation: Varied clinical presentation from infancy through adulthood. Surgery. $2011 ; 149(3): 386-93$. doi: 10.1016/j.surg.2010.07.004.

10. Ayantunde AA, Agrawal A. Gallstone Ileus: Diagnosis and Management. World J Surg. 2007;31(6):1294-9. doi: 10.1007/s00268-007-9011-9.

11. Day EA, Marks C. Gallstone ileus. Am J Surg. 1975;129(5):552-8. doi: 10.1016/0002-9610(75)90315-3.

12. Kasahara Y, Umemura H, Shiraha S. Gallstone ileus: review of 112 patients in the Japanese literature. Am J Surg. $1980 ; 140(3): 437-40$. doi: 10.1016/0002-9610(80)90185-3.

13. Lassandro F, Gagliardi N, Scuderi M, Pinto A, Gatta G, Mazzeo R. Gallstone ileus analysis of radiological findings in 27 patients. Eur J Radiol. 2004;50(1):23-9.

doi: 10.1016/j.ejrad.2003.11.011.

14. Reisner RM, Cohen JR. Gallstone ileus: a review of 1001 reported cases. Am Surg. 1994;60(6):441-6.

15. Rodríguez-Sanjuán JC, Casado F, Fernández MJ, Morales DJ, Naranjo A. Cholecystectomy and fistula closure versus enterolithotomy alone in gallstone ileus. $\mathrm{Br} \mathrm{J}$ Surg. 1997;84(5):634-7.

16. Zuegel N, Hehl A, Lindemann F, Witte $\mathrm{J}$. Advantages of one-stage repair in case of gallstone ileus. Hepatogastroenterology. 1997;44(13):59-62.

17. Dietz DW, Walsh RM, GrundfestBroniatowski S, Lavery IC, Fazio VW, Vogt DP. Intestinal malrotation: a rare but important cause of bowel obstruction in adults. Dis Colon Rectum. 
2002;45(10):1381-6.

doi: 10.1097/01.dcr.0000029637.48119.8D.

18. Ladd WE. Surgical Diseases of the Alimentary Tract in Infants. N Engl $\mathrm{J}$ Med. 1936;215(16):705-8. doi: $10.1056 /$ NEJM193610152151604.

19. Choi M, Borenstein SH, Hornberger L, Langer JC. Heterotaxia syndrome: the role of screening for intestinal rotation abnormalities. Arch Dis Child. 2005;90(8):813-815.

doi: $10.1136 /$ adc.2004.067504.
Fonte de financiamento: Não

Conflito de interesses: Não

Data de Submissão: 24 Março 2021

Decisão final: 02 Setembro 2021

\section{Autor de Correspondência:}

Henrique Cunha Mateus

E-mail: hcmateus@gmail.com

b.hirata@hotmail.com 